\title{
APROXIMAÇÃO EXTERNA/DECOMPOSIÇÃO DE BENDERS PARA PROJETO DE REDES SOB CONGESTIONAMENTO VIA $\lambda$-ÓTIMO
}

\author{
Karolliny Danielle Santos \\ Universidade Federal de Minas Gerais \\ UFMG - Programa de Pós Graduação em Engenharia de Produção \\ Av. Antônio Carlos, 6627 - Pampulha \\ CEP: 31270-901 - Belo Horizonte - Minas Gerais - Brasil \\ karollinny.ds@gmail.com \\ Gilberto de Miranda Júnior \\ Universidade Federal de Minas Gerai \\ UFMG - Programa de Pós Graduação em Engenharia de Produção \\ Av. Antônio Carlos, 6627 - Pampulha \\ CEP: 31270-901 - Belo Horizonte - Minas Gerais - Brasil \\ miranda@dep.ufmg.br \\ Ricardo Saraiva de Camargo \\ Universidade Federal de Minas Gerai \\ UFMG - Programa de Pós Graduação em Engenharia de Produção \\ Av. Antônio Carlos, 6627 - Pampulha \\ CEP: 31270-901 - Belo Horizonte - Minas Gerais - Brasil \\ rcamargoddep.ufmg.br
}

\begin{abstract}
RESUMO
Neste trabalho apresenta-se a técnica de seleção de cortes de Benders conhecida como $\lambda$-ótimo ao problema de projeto de redes sob congestionamento, via Aproximação Externa/ Decomposicão de Benders. O problema de projeto de redes tem uma ampla aplicação em atividades de planejamento, estratégias de empresas que operam sistemas de distribuição, produção, transporte de energia, matéria, etc; visa atender as demandas dos clientes, assegurar o lucro e a eficiência da operação e respeitar os níveis de serviço pré-estabelecidos. Deste esforço de modelagem derivou um programa não-linear, NP-difícil e de grande porte. Experimentos computacionais e comparações com versões mais simples demonstram o sucesso do esquema adotado.

Palavras chave: Projeto de Redes com Custos Convexos; Método de Decomposição de Benders; Aproximação Externa; Esquema $\lambda$-ótimo.
\end{abstract}

\begin{abstract}
This work presents the Benders selection cuts technique known as Optimal-lambda to the network congestion project problem, which uses Outer approximation / Benders decomposition. The network project problem has a wide application in planning activities, business strategies that operate at distribution systems, production, energy transport, material, etc; proposes attend the customer demand, ensure profit and efficiency of the operation and respect the pre-established service levels. This modeling derived a nonlinear program, which is a large scale NP-hard. Computational experiments and comparisons with standard versions demonstrate the efficiency of the proposed approach.
\end{abstract}

Keywords: Convex network design; Benders decomposition; Outer approximation; Optimal-lambda scheme. 


\section{Introdução}

As redes são as veias entre todas as atividades de comunicação espalhadas pelo espaço e tempo. A variedade de tipos de redes inclui os sistemas de distribuição de energia, fornecimento de água, serviços de saúde, malhas ferroviarias e rodoviárias, sistemas de estradas, redes de telecomunicações, etc. As redes de energia fornecem a alimentação para execução de atividades economicas; redes de transporte possibilitam a movimentação de pessoas, bens e serviços; redes de comunicação permitem a troca de informações e mensagem.

Dado um grafo, o problema de projeto de redes consiste em encontrar um subgrafo que liga um nó fornecedor a um nó de demanda a um custo mínimo. O principal objetivo do problema é atender as demandas dos clientes, assegurar o lucro e a eficiência da operação e respeitar os níveis de serviço pré-estabelecidos. Deve-se considerar tanto a adequação dos níveis de serviços da infraestrutura afim de manter o nível de serviço necessário quanto a necessidade de operação otimizada que racionalize o uso da capacidade instalada. Segundo Dias et al. (2011) demonstrar um bom comportamento no que diz respeito às duas versões compreende investir mais em infra-estrutura e aumentar a capacidade instalada ou priorizar a otimização das práticas operacionais, buscar o equilíbrio entre essas versões é a raiz de uma estratégia bem sucedida, uma forma de tal equilíbrio é estudar o problema sob a perspectiva da Programação Matemática.

Conforme Maculan (1987) este problema pode ser visto, do ponto de vista matemático, como uma extensão do Problema da Árvore de Steiner em um grafo dirigido. De acordo com Maculan (1987) e Hwang and Richards (1992) desprezando os custos de transmissão e de serviço nas conexões, um Problema de Steiner é gerado, enquanto para Dantzig (1962) um problema de transbordo de fonte única é obtido quando desconsidera-se os custos fixos.

Um dos principais trabalhos abordando projeto de redes com congestionamento foi proposto por Miranda et al. (2011), no qual considera uma formulação de multimercadoria que é capaz de lidar com o projeto de redes de computadores centralizados, multi-cast, multi-party ou árvores de transporte baseados em hub. Dias et al. (2011) aborda o mesmo problema resolvendo-o por meio do Método de Decomposição de Benders Generalizado e Aproximação Externa; o problema consiste basicamente em ligar um servidor aos seus diversos clientes dispersos geograficamente, satisfazendo a demanda a um custo mínimo total.

As várias aplicações do problema em estudo são vistas nas áreas de distribuição de energia elétrica como em Dantzig (1962) e Ramírez-Rosado and Domínguez-Navarro (2006), de transporte de cargas fracionadas (Cordeau et al. (2006)), de transporte público (Contreras et al. (2009); Contreras et al. (2010)), de rede de acesso local de telefonia (Randazzo and Luna (2001)), e de redes de computadores (Klincewicz (1998), Altiparmak et al. (2003)). Um excelente survey envolvendo diferentes variações do problema e de suas técnicas de solução pode ser encontrado em (Costa (2005)).

Neste trabalho problemas de projeto de redes congestionadas são abordados. Apresenta-se a extensão dos trabalhos de Miranda et al. (2011) e Dias et al. (2011) onde considera-se o problema de projeto de redes sob congestionamento apresentando técnicas de seleção de cortes de Benders conhecida como $\lambda$-ótimo atacado via Aproximação Externa/Decomposição de Benders (OA+BD). No problema associa-se a cada arco da rede três custos: custo fixo que representa a conexão do arco, um custo operacional unitário de transmissão de uma unidade de fluxo através do arco e um custo de congestionamento não linear. Além disso, o problema pode ter terminais heterogêneos, como o nó de Steiner ou de transbordo. Apresenta-se redes arborescentes de fonte única com custos convexos, considerando custos de instalação, infra-estrutura, transporte e congestionamento. Esses últimos são responsáveis pela degradação dos níveis de serviço e economia de escala em cada arco.

Em termos gerais as técnicas propostas aqui podem ser empregadas em problemas reais de projeto de rede de distribuição de energia ou de redes de dados, no entanto, este modelo descreve melhor problemas de redes de transportes de informação; para estudos posteriores pode-se fazer as adequações necessárias, na linha proposta por Benchakroun et al. (1992) onde é possivel abordar 
problemas de projeto de redes de transmissão de energia que envolvam funções sofisticadas, desde que o esforço de convexificação garanta boa presição na descrição dos efeitos não-lineares.

Formulações matemáticas são desenvolvidas para projeto de redes sob congestionamento. Até hoje tais problemas não podem ser resolvidos eficientemente, sendo assim, a principal contribuição deste trabalho concentra-se na resolução de um problema não-linear, NP-difícil e de grande porte de forma eficiente. Estabelecida a relevância de tais problemas, desenvolve-se um eficiente algoritmo para resolver o problema. Utiliza-se técnicas de Decomposição de Benders (BD) e o método de Aproximação Externa $(\mathrm{OA})$, além da técnica $\lambda$-ótimo para acelerar a convergência dos métodos, está combinação permitiu reduzir o tempo computacional de resolução e aumentar o tamanho da redes usadas como teste. Resultados experimentais confirmam a eficiência deste algoritmo ao resolver instâncias de grande porte.

O trabalho está assim organizado: na Seção 2, são apresentadas as definições usadas e a formulação proposta. Na Seção 3: Metodos de resolução: A decomposição de Benders e a Aproximação Externa são formalizadas. Na Seção 4: Apresenta-se alguns metodos de aceleração de convergência do algoritmo. Por fim, os experimentos computacionais e os comentários finais são feitos nas Seções 5 e 6 respectivamente.

\section{Definições e Formulação}

Uma rede genérica é representada por grafo direcionado $\Gamma(V, E)$, onde $\mathrm{V}$ e $\mathrm{E}$ são os conjuntos de nós e arcos, que representam as possíveis conexões. Um arco ou ligação entre os nós $i$ e $j$ é representado por $(i, j) \in E$, onde $i, j \in V$, e o índice $k \in K$. Seja $s$ é o nó fonte, que deve enviar a energia, matéria ou informação a $\mathrm{K}$ de nós de demanda $d_{k}$, e $K$ é o conjunto de nós de demanda, onde $K \subseteq V$.

O custo unitário de transporte/transmissão da demanda no nó $k \in K$ no $\operatorname{arco}(i, j) \in$ $E$ é representado por $c_{i j k}$. Assume-se $c_{i j k}=\theta_{i j}^{k} \delta_{i j}$, onde $\theta^{k}$ é o custo específico de transporte de demanda do nó $k$, e $\delta_{i j}^{k}$ é a distância entre os nós $i$ e $j$, o custo de ativação do arco, $b_{i j}$, onde $b_{i j}=\zeta_{i j} \delta_{i j}, \forall(i, j) \in E$ e $\zeta_{i j}$ é custo da instalação da infraestrutura por unidade de distância. Utiliza-se as seguintes variáveis de decisão: $x_{i j} \in\{0,1\}, \forall(i, j) \in E$, que indica se o arco $(i, j)$ é ativado $\left(x_{i j}=1\right)$ ou não $\left(x_{i j}=0\right) ; f_{i j k} \geq 0$ : representa o fluxo com destino a $k \in K$ passando pelo $\operatorname{arco}(i, j) \in E$ e $g_{i j} \geq 0$ representa o fluxo global do $\operatorname{arco}(i, j) \in E$.

A função convexa não-linear de degradação do nível de serviço é representada pelo custo de congestionamento nos arcos, sendo separável por arco e representada pela seguinte lei de potência:

$$
\tau_{i j}\left(g_{i j}\right)=e g_{i j}^{p}, \quad \forall(i, j) \in E
$$

$e>0$ e $p \geq 1$ são escalares que representam a participação dos custos de congestionamento do custo total.

Em geral, o problema de projeto de redes com congestionamento pode ser formulado como:

$$
\begin{aligned}
& \min \sum_{(i, j) \in E}\left[b_{i j} x_{i j}+\tau_{i j}\left(g_{i j}\right)+\sum_{k \in K} c_{i j k} f_{i j k}\right] \\
& \text { s.t.: } \sum_{i \in V} x_{i j} \leq 1 \quad \forall j \in V \\
& \sum_{k \in K} f_{i j k}-g_{i j}=0 \quad \forall(i, j) \in E \\
& \sum_{(s, j) \in E} f_{s j k}=d_{k} \quad \forall k \in K
\end{aligned}
$$




$$
\begin{aligned}
& \sum_{(i, k) \in E} f_{i k k}=d_{k} \\
& \sum_{(i, j) \in E} f_{i j k}=\sum_{(j, i) \in E} f_{j i k} \\
& f_{i j k} \leq d_{k} x_{i j} \\
& f_{i j k} \geq 0 \\
& g_{i j} \geq 0 \\
& x_{i j} \in\{0,1\}
\end{aligned}
$$

$$
\begin{aligned}
& \forall k \in K \\
& \forall j \in V \backslash S, k \in K: k \neq j \\
& \forall(i, j) \in E, k \in K \\
& \forall(i, j) \in E, k \in K \\
& \forall(i, j) \in E \\
& \forall(i, j) \in E
\end{aligned}
$$

A função objetivo (2) possui 3 termos: O primeiro termo contabiliza o custo total da instalação dos arcos; o segundo calcula os custos convexos de congestionamento; enquanto o terceiro totaliza o custo de transporte das diversas demandas. As restrições (3) obrigam a geração de arborescencias ao impedir a incidência de mais de um arco em cada nó. As restrições (4) calculam o fluxo global que atravessa o arco $(i, j)$. As restrições (5)- (7) garantem o balanço de fluxo para cada produto em cada nó desde a origem $s$ até cada nó de destino $k$. As restrições de aclopamento (8) permintem que o fluxo passe através do arco $(i, j)$ somente se o mesmo estiver instalado. Finalmente (9) e (10) são as restrições de não negatividade para os fluxos $f_{i j k}$ e $g_{i j}$ respectivamente, enquanto as restrições (11) obrigam a integralidade das variavéis $x_{i j}$.

\section{Método de Resolução}

Ao abordar problema de PNLIM, destacam-se dois métodos de grande escala para a resolução desses problemas: A Decomposição de Benders (Geoffrion (1972)) e a Aproximação Externa ( Duran and Grossmann (1986), Fletcher and Leyffer (1994) e Yuan et al. (1988)). Ambos idealizam decompor o problema original em dois níveis: no nível superior, conhecido como problema mestre (PLIM), onde é resolvido um problema inteiro misto e no nível inferior, conhecido como subproblema (SP), resolve-se um subproblema não linear, o problema é resolvido de forma iterativa até que o limite inferior (LI) convirja para o limite superior (LS). A diferença entre os dois métodos é que o BD exclui um conjunto de variáveis fracionárias, pertencentes ao problema original, do PM, enquanto o OA mantém todas as variáveis do problema original no PM. Sendo assim, ao tratar a formulação com limites inferiores o PM-OA fornece limites inferiores maiores ou iguais aos obtidos pelo BD, implicando em menores iterações para a convergência, entretanto como o PM-OA é um pouco maior tornando-o mais difícil de ser resolvido, pois possui um número bem maior de variavéis e restrições, veja (Duran and Grossmann (1986)).

\subsection{Decomposicão de Benders}

Considere o seguinte problema de PLIM:

$$
\min \left\{c^{T} x+f^{T} y: A x \geq b ; D x+B y \geq d ; x \in Z_{+}^{n} ; y \in \Re_{+}\right\}
$$

Introduz-se uma variavél artificial $\eta=f^{T} y$, com um limite $\bar{\eta}$ e o Problema Mestre Relaxado (PMR):

$$
\min \left\{c^{T} x+\eta: A x \geq b ; \eta \geq \bar{\eta} ; x \in Z_{+}^{n}\right\}
$$

O problema é resolvido de forma iterativa. Uma solução ótima encontrada $\left(x^{*}, \eta^{*}\right) \operatorname{com} x^{*} \in Z$ é enviado para o SP:

$$
\max \left\{\pi^{T}\left(d-D x^{*}\right): \pi^{T} B \leq f^{T}, \pi \geq 0\right\}
$$

Se o SP é ilimitado, um raio extremo $\pi^{T}$ é escolhido e o Corte de Viabilidade de Benders é adicionado ao PM $\overline{\pi^{T}}\left(d-D x^{*}\right) \leq 0$ onde é novamente resolvido. Caso contrário, assume $z^{*}$ e $\bar{\pi}$ indica o valor ótimo do SP, respectivamente. Se $z^{*} \leq \eta^{*}$, então $\left(x^{*}, \eta^{*}\right)$ é viável e esses são ótimos para (12). Se não, o Corte de Otimalidade de Benders $\eta \geq \overline{\pi^{T}}\left(d-D x^{*}\right)$ é adicionado ao PM e o que segue é resolvido novamente. Aplicando o método ao problema proposto temos a formulação mestre: 


\subsection{O Problema Mestre Relaxado}

Algumas vezes, a solução encontrada pelo PMR pode acarretar em ciclos na topologia proposta ou em sub-árvore desconexas. Logo, trabalha - se com ambos os conjuntos de variáveis de x e g no problema mestre relaxado. A ideia aqui é garantir o fechamento de balanço de fluxos no nível superior, evitando a geração de topologias inviáveis. Portanto temos o seguinte PMR:

$$
\begin{aligned}
& \min _{t, x \in X} \sum_{(i, j) \in E} b_{i j}\left(x_{i j}\right)+\sum_{k} t_{k} \\
& \text { s.t.: }(10)-(11) \\
& \sum_{i \in V} x_{i j} \leq 1 \\
& \sum_{(i, k) \in E} g_{i k}-\sum_{(k, j) \in E} g_{k j}=d_{k} \\
& \sum_{(i, j) \in E} g_{i j}-\sum_{(j, i) \in E} g_{j i}=0 \\
& \sum_{(s, j) \in E} g_{s j}=\sum_{k \in K} d_{k} \\
& g_{i j} \leq \sum_{k \in K} d_{k} x_{i j} \\
& t_{k} \geq \nu_{k}\left(x^{h}\right)+\sum_{i j} \alpha_{i j k}^{h} d_{k}\left(x_{i j}^{h}-x_{i j}\right) \quad \forall k \in K \\
& t \geq 0
\end{aligned}
$$

$\left.\alpha_{i j k}^{h}\right) f_{i j k}$.

Onde $\nu_{k}\left(x^{h}\right)=\sum_{(i, j) \in E} \sum_{k \in K}-\alpha_{i j k}^{h} d_{k} x_{i j}+\min \sum_{(i, j) \in E} \tau_{i j} g_{i j}+\sum_{k \in K}\left(c_{i j k}+\right.$

As variáveis (17)- (19) são restrições de balanço de fluxo no espaço das variáveis $g$, ao utilizá-las, evita-se uso dos cortes de Benders do tipo II, ou seja, cortes associados a raios extremos do poliedro original (2)- (11). Caso contrário, gastaria-se tempo para resolver problemas inteiros apenas para descartar configurações de redes inviáveis.

\subsection{Subproblema Linear: Primal e Dual}

Para x fixado em $x^{h}$ o subproblema primal seria:

$$
\begin{aligned}
& \min \sum_{(i, j) \in E} c_{i j k} f_{i j} \\
& \text { s.t.: } \sum_{(s, j) \in E} f_{s j k}=d_{k} \\
& \sum_{(i, k) \in E} f_{i k k}=d_{k} \\
& \sum_{(i, j) \in E} f_{i j k}=\sum_{(j, i) \in E} f_{j i k} \\
& -f_{i j k} \geq-d_{k} x_{i j} \quad \forall(i, j) \in E \\
& f_{i j k} \geq 0
\end{aligned}
$$

É sabidamente mais útil trabalhar com a versão dual de (24)- (29). Associando as variáveis $p_{j k}, p_{s k}, p_{k k}$ e $\alpha_{i j k}$. Obtém-se o respectivo subproblema dual, para cada produto k:

$$
\max _{p, \alpha \geq 0} d_{k}\left(p_{k k}-p_{s k}-\sum_{(i, j) \in E} x_{i j} \alpha_{i j k}\right)
$$




$$
p_{j k}-p_{i k}-\alpha_{i j k} \leq c_{i j k} \quad \forall(i, j) \in E
$$

Enquanto o SP primal tem solução única o SP dual pode ter multiplas soluções ótimas tornando a escolha das variáveis duais ótimas um tópico a parte.

\subsection{Aproximação Externa}

A técnica conhecida como Aproximação Externa(OA) (do inglês Outer Approximation) foi desenvolvida em trabalhos pioneiros de Duran and Grossmann (1986), Fletcher and Leyffer (1994) e Yuan et al. (1988). Tem sido aplicada em otimização de síntese de processos (Grossmann and Kravanja (1995)), (Karuppiah et al. (2008)), e em várias outras aplicações de projeto em engenharia. Mais recentemente, o método tem sido aplicado a sistemas logísticos como em Huang et al. (2005) e em problemas de otimização em geral ( Grossmann and Kravanja (1995)).

$\mathrm{Na}$ formulação (2)- (11), $\left(\sum_{(i, j) \in E}\left(\tau_{i j} g_{i j}\right)\right)$ é o único termo não-linear. Portanto, esse é o termo que necessita sofrer aproximação externa. Pode-se substituir $\tau_{k}\left(g_{k}\right)$ por $\xi_{i j}$ para cada $k$ na função objetivo.

A ideia aqui é permitir a solução do problema mestre OA, por meio de algoritmo de decomposição de Benders. Lida-se com a não-linearidade via OA e com a parte de grande escala via Decomposição de Benders.

$$
\begin{aligned}
& \min \sum_{(i, j) \in E}\left[b_{i j} x_{i j}+\xi_{i j}+\sum_{k} t_{k}\right] \\
& \text { s.t.: }(10)-(11),(16)-(19) \\
& \xi_{i j} \geq \tau_{i j}\left(g_{i j}^{h}\right)+\beta_{i j}^{h}\left(g_{i j}-g_{i j}^{h}\right) \\
& \forall(i, j) \in E, \quad \forall h=1, . ., H \\
& t_{k} \geq \nu_{k}\left(x^{l}\right)+\sum_{i j} \alpha_{i j k}^{l} d_{k}\left(x_{i j}^{l}-x_{i j}\right) \\
& \xi_{i j} \geq 0 \\
& t_{k} \geq 0 \\
& \text { (34) } \\
& \forall k \in K \\
& \forall(i, j) \in E \\
& \forall k \in K
\end{aligned}
$$

O uso concorrente de Aproximação Externa e Decomposição de Benders (OA+BD) já foi aplicado com sucesso para problemas de projeto de redes (Dias et al. (2011)), de localização de hubs (De Camargo et al. (2011)).

Magnanti and Wong (1981) introduziram novas técnicas para acelerar a convergência do método de Decomposição de Benders. A técnica de aceleração é baseada em uma seleção criteriosa das variáveis duais que sejam Pareto-ótimas.

\section{Técnicas de Aceleração}

Magnanti and Wong (1981) notaram que quando o SP é degenerado, exite um conjunto de diferentes cortes que poderiam ser gerados. Portanto, a ideia principal da técnica de Magnanti e Wong consiste em adicionar ao PM a cada iteração cortes Pareto-ótimo, ou seja, aqueles que não são dominados por nenhum outro corte. Para gerar cortes Pareto-ótimo Magnanti e Wong utilizam um conjunto de pontos auxiliares, denominados core-points. Um ponto é denominado core-point se ele pertence ao interior da casca convexa dos pontos interiores viáveis.

Seja $\pi_{h}$ e $f\left(\pi_{h}\right)$, a solução ótima e o valor da função objetivo do SP associado a solução $x_{h}$ do PM e seja $x^{0}$ um core-point válido na iteração h. Logo temos o respectivo Subproblema de Magnanti e Wong (SMW) para gerar cortes Pareto-ótimo:

$$
\begin{aligned}
& \max \left(d-D x^{0}\right)^{T} \pi \\
& \text { s.t.: }\left(d-D x_{h}\right)^{T} \pi=f^{T}\left(\pi_{h}\right)
\end{aligned}
$$




$$
\begin{array}{r}
B^{T} \pi \leq f^{T} \\
\pi \geq 0
\end{array}
$$

A equação (39), entretanto, insere instabilidade numérica no SP dual, o que torna o uso prático de método de Magnanti and Wong (1981) limitado. Papadakos (2008) propõe remover a equação (39) e substituí-la por uma atualização em vôo do core-point, removendo então os problemas de natureza numérica, veja (42) e (43).

$$
\begin{aligned}
& x_{h+1}^{0}=(1-\lambda) x_{h}^{0}+\lambda x_{h} \\
& 0 \leq \lambda \leq 1
\end{aligned}
$$

Papadakos propõe ainda relaxar o conceito de core-point, bastando garantir a validade de seu Teorema 7 para gerar cortes Pareto-ótimo mesmo que fora do interior relativo da casca convexa de soluções inteiras viáveis, vide o Teorema 7 em Papadakos (2008).

Uma extensão das ideias de Papadakos permite gerar cortes Pareto-ótimo a partir de soluções em que o SP é inviável. Essa modificação aumenta a velocidade de recuperação de informação do subproblema e permite utilizar a parte útil da solução do PM, em vez de meramente gerar um corte de viabilidade. Há também que se considerar que diversas soluções inviáveis sejam topologicamente similares à solução ótima.

Para tanto, basta escrever um problema auxiliar no peso $\lambda$ usado na equação (44):

$$
\begin{array}{ll} 
& \max \lambda_{k} \\
& \sum_{j \backslash(s, j) \in E} f_{s j k}=d_{k} \\
& \sum_{j \backslash(j, k) \in E} f_{j k k}=d_{k} \\
& \sum_{j \backslash(j, i) \in E} f_{j i k}=\sum_{j \backslash(i, j) \in E} f_{i j k} \\
f_{i j k} \leq d_{k}\left[(1-\lambda) x_{i j}^{0}-\lambda_{k} x_{i j}^{h}\right] & \\
f_{i j k}^{h} \geq 0 &
\end{array}
$$

Para cada produto k. Naturalmente ao atualizar o core-point, usa-se $\lambda$ tal que $\lambda=$ $\min _{k}\left\{\lambda_{k}\right\}$, e procede-se à geração de um corte de otimalidade Pareto-ótimo. Essa técnica já foi aplicada com grande sucesso em outro problema, veja (de Sá et al. (2013)).

\section{Experimentos Computacionais}

Os testes computacionais foram realizados utilizando um sistema operacional Linux 64 bits e os experimentos feitos em uma estação de trabalho com o processador Intel(R) Xeon $(R) C P U$ E5630 com 2.53GHz e $24 G B$ de memória RAM. Códigos foram implementados em Concert/Cplex usando o CPLEX. Para essas formulações utiliza-se um conjunto de instâncias disponíveis na biblioteca online QAPLIB ( Hahn et al. (2006)). O número de nós e os tamanhos dos conjuntos E e K são mostrados na tabela (1).

Para cada conjunto de testes foram analisados o tempo computacional gasto e o gap de otimalidade. Para gerar a primeira solução viável são utilizados a Árvore Geradora Mínima (AGM) e a Árvore de Caminho Mínimo (ACM). Dois conjuntos de experimentos usando instâncias padrão da literatura foram realizados afim de avaliar a eficiência dos algoritmos propostos. Esses conjuntos 
Tabela 1: Análise das instâncias em relação ao tamanho dos conjuntos.

\begin{tabular}{cccc}
\hline \hline Instância & $|\mathrm{E}|$ & $|\mathrm{K}|$ & $|\mathrm{V}|$ \\
\hline 14 & 182 & 9 & 14 \\
15 & 210 & 9 & 15 \\
17 & 272 & 10 & 17 \\
18 & 306 & 11 & 18 \\
20 & 380 & 17 & 20 \\
21 & 420 & 14 & 21 \\
22 & 462 & 14 & 22 \\
24 & 552 & 16 & 24 \\
25 & 600 & 17 & 25 \\
27 & 702 & 18 & 27 \\
28 & 756 & 19 & 28 \\
30 & 870 & 21 & 30 \\
40 & 1560 & 25 & 40 \\
50 & 2450 & 31 & 50 \\
60 & 3540 & 36 & 60 \\
80 & 6320 & 48 & 80 \\
100 & 9900 & 59 & 100 \\
150 & 22350 & 92 & 150 \\
\hline \hline
\end{tabular}

de experimentos foram dividido em duas fases. A primeira fase tem o objetivo de avaliar qual é a melhor variante do método $\mathrm{OA}+\mathrm{BD}$ ao combinar diferentes estratégias para acelerar a convergência do método, enquanto a segunda fase consiste em comparar a melhor variante encontrada com o CPLEX.

Nesta primeira fase faz-se comparações entre os algoritmos Cplex, $O A+B D$, Agregado, Desagregado e $\lambda$-Ótimo.

- Cplex: Formulação Original.

- OA+BD: Algoritmo utilizando os métodos de Decomposicão de Benders e o Aproximação Externa.

- Agregado: Além da utilização do (OA+BD), utiliza-se ainda os cortes Callbacks adicionados em uma única árvore.

- Desagregado: Utiliza-se o $(\mathrm{OA}+\mathrm{BD})$, os cortes callbacks adicionando os cortes de viabilidade e também os de otimalidade.

- $\lambda$-Ótimo: É uma versão da junção de todos os algoritmos citados anteriormente e a utilização do $\lambda$-ótimo.

As tabelas (2) e (3) demostram que a variante $\lambda$-ótimo apresenta os melhores desempenhos ao considerar o problema com congestionamento e principalmente a medida que as instâncias aumentam.

Em média esta técnica se mostra muito eficiente tornando o método até 37 vezes mais rápido ao comparar com o tempo gasto pelo CPLEX, como pode-se ver na instância 20 nós entre outras. Pode-se ver, que os cortes de Benders agregado são bastante competitivos, mas medida a que a instância aumenta o tempo computacional aumenta tornando assim mais adequado utilizar o problema desagregados. 
Tabela 2: Comparativo entre os algoritmos Cplex , $O A+B D$, Agregado, Desagregado e o algoritmo $\lambda$-ótimo em relação ao tempo gasto.

\begin{tabular}{|c|c|c|c|c|c|c|}
\hline Instância & ex & $\begin{array}{c}\text { Cplex } \\
\text { T(s) }\end{array}$ & $\begin{array}{c}\mathbf{O A}+\mathbf{B D} \\
\mathbf{T}(\mathbf{s})\end{array}$ & $\begin{array}{c}\text { Agregado } \\
\text { T(s) }\end{array}$ & $\begin{array}{c}\text { Desagregado } \\
\mathbf{T}(\mathbf{s})\end{array}$ & $\begin{array}{c}\lambda \text {-ótimo } \\
\mathbf{T}(\mathbf{s})\end{array}$ \\
\hline \multirow{3}{*}{12} & 0.0 & 0.0300 & 0.0400 & 0.0600 & 0.0500 & 0.0500 \\
\hline & 0.01 & 0.3200 & 0.3200 & 0.1300 & 0.1900 & 0.1900 \\
\hline & 0.05 & 1.7800 & 1.8200 & 0.7300 & 0.9600 & 1.1500 \\
\hline \multirow{3}{*}{14} & 0.0 & 0.0300 & 0.0800 & 0.1000 & 0.1000 & 0.1100 \\
\hline & 0.01 & 0.6000 & 0.6600 & 0.3400 & 0.5200 & 0.3900 \\
\hline & 0.05 & 4.5200 & 2.7000 & 0.5700 & 1.2300 & 0.9800 \\
\hline \multirow{3}{*}{15} & 0.0 & 0.0400 & 0.0900 & 0.0900 & 0.0800 & 0.1100 \\
\hline & 0.01 & 0.5100 & 0.6800 & 0.3000 & 0.4400 & 0.3300 \\
\hline & 0.05 & 5.6500 & 2.2400 & 0.5100 & 0.6400 & 0.6200 \\
\hline \multirow{3}{*}{17} & 0.0 & 0.0500 & 0.0600 & 0.1300 & 0.0700 & 0.1800 \\
\hline & 0.01 & 1.0700 & 1.2900 & 0.3800 & 0.7000 & 0.3500 \\
\hline & 0.05 & 10.4300 & 4.5900 & 0.6600 & 0.9700 & 1.1900 \\
\hline \multirow{3}{*}{18} & 0.0 & 0.0700 & 0.1000 & 0.2000 & 0.1200 & 0.2100 \\
\hline & 0.01 & 1.7000 & 2.3600 & 0.7300 & 1.6400 & 0.9400 \\
\hline & 0.05 & 38.2200 & 20.5900 & 4.0600 & 7.5400 & 5.6700 \\
\hline \multirow{3}{*}{20} & 0.0 & 0.1200 & 0.1000 & 0.2900 & 0.1800 & 0.5200 \\
\hline & 0.01 & 16.4400 & 24.7700 & 6.4100 & 5.6600 & 6.5400 \\
\hline & 0.05 & 1415.4000 & 259.2500 & 21.1700 & 73.7000 & 38.2900 \\
\hline \multirow{3}{*}{22} & 0.0 & 0.1300 & 0.1600 & 0.4900 & 0.4500 & 0.5900 \\
\hline & 0.01 & 88.8400 & 62.8200 & 21.5400 & 26.7100 & 35.4300 \\
\hline & 0.05 & 3600.000 & 3600.000 & 3600.000 & 3600.000 & 2462.820 \\
\hline \multirow{3}{*}{24} & 0.0 & 0.2100 & 0.3800 & 0.8200 & 0.6000 & 1.0200 \\
\hline & 0.01 & 684.5000 & 376.9000 & 1233.4800 & 250.6800 & 233.4800 \\
\hline & 0.05 & 3600.000 & 3600.000 & 3600.000 & 3600.000 & 3600.000 \\
\hline \multirow{3}{*}{25} & 0.0 & 0.2100 & 0.2900 & 1.0200 & 0.3400 & 1.6500 \\
\hline & 0.01 & 1913.6800 & 839.7700 & 234.0100 & 726.7200 & 334.6600 \\
\hline & 0.05 & 3600.000 & 3600.000 & 3600.000 & 3600.000 & 3600.000 \\
\hline \multirow{3}{*}{27} & 0.0 & 0.2600 & 0.4200 & 2.1200 & 0.7100 & 1.8200 \\
\hline & 0.01 & 3600.000 & 3600.000 & 3600.000 & 3600.000 & 3600.000 \\
\hline & 0.05 & 3600.000 & 3600.000 & 3600.000 & 3600.000 & 3600.000 \\
\hline \multirow{3}{*}{28} & 0.0 & 0.3000 & 0.2700 & 3.7600 & 0.8000 & 2.7300 \\
\hline & 0.01 & 164.8900 & 45.0500 & 14.3200 & 41.6200 & 22.9800 \\
\hline & 0.05 & 3600.000 & 3600.000 & 3600.000 & 3600.000 & 3600.000 \\
\hline \multirow{3}{*}{30} & 0.0 & 0.4800 & 3.1400 & 3.1800 & 2.5900 & 4.0000 \\
\hline & 0.01 & 335.0500 & 73.8300 & 14.0900 & 14.1400 & 24.6700 \\
\hline & 0.05 & 3600.000 & 3600.000 & 3600.000 & 3600.000 & 2459.3600 \\
\hline
\end{tabular}

Tabela 3: Analise das instâncias que não obtiveram o gap de otimalidade como o valor $=0$, para as demais instâncias tem-se o gap $=0.0$.

\begin{tabular}{ccccccc}
\hline \hline Instância & ex & $\begin{array}{c}\text { Cplex } \\
\text { GAP(\%) }\end{array}$ & $\begin{array}{c}\text { OA+BD } \\
\text { GAP(\%) }\end{array}$ & $\begin{array}{c}\text { Callback } \\
\text { GAP(\%) }\end{array}$ & $\begin{array}{c}\text { Callback multi-cuts } \\
\text { GAP(\%) }\end{array}$ & $\begin{array}{c}\lambda \text {-ótimo } \\
\text { GAP(\%) }\end{array}$ \\
\hline 22 & 0.01 & 0.0 & 0.0 & 0.0 & 0.0 & 0.0 \\
& 0.05 & 0.64 & 0.0173 & 1.66 & 3.3 & 0.0 \\
\hline 24 & 0.01 & 0.0 & 0.0 & 0.0 & 0.0 & 0.0 \\
& 0.05 & 3.72 & 0.8091 & 2.61 & 2.89 & 1.80 \\
\hline 25 & 0.01 & 0.0 & 0.0 & 0.0 & 0.0 & 0.0 \\
& 0.05 & 4.40 & 4.6413 & 1.62 & 3.40 & 1.63 \\
\hline 27 & 0.01 & 2.20 & 1.0216 & 1.29 & 3.39 & 1.04 \\
& 0.05 & 7.26 & 13.4597 & 6.38 & 7.31 & 4.85 \\
\hline 28 & 0.01 & 0.0 & 0.0 & 0.0 & 0.0 & 0.0 \\
& 0.05 & 2.78 & 1.1493 & 1.97 & 5.79 & 1.50 \\
\hline 30 & 0.01 & 0.0 & 0.0 & 0.0 & 0.0 & 0.0 \\
& 0.05 & 2.03 & 2.6155 & 1.19 & 1.50 & 0.0 \\
\hline \hline
\end{tabular}


Para a segunda fase de testes faz-se comparações entre os algoritmos Cplex, agregado e desagregado:

- Cplex: Formulação Original utilizando o CPLEX 12.3.

- Agregado: Algoritmo (OA+BD) via $\lambda$-ótimo que gera um único corte por iteração.

- Desagregado: O algoritmo (OA+BD) via $\lambda$-ótimo que gera multiplos cortes por cada iteração, ou seja, gera um corte para cada $k$ a cada iteração.

Tabela 4: Comparativo entre os algoritmos Cplex, Agregado e o Desagregado em relação ao tempo gasto.

\begin{tabular}{|c|c|c|c|c|c|}
\hline Instância & $\tau$ & $\begin{array}{c}\text { Cplex } \\
\text { T(s) }\end{array}$ & $\begin{array}{c}\text { Agregado } \\
\text { T(s) }\end{array}$ & $\begin{array}{c}\text { Desagregado } \\
\text { T(s) }\end{array}$ & $\overline{\text { Capacidade }}$ \\
\hline \multirow{2}{*}{15} & 10 & 0.0200 & 0.0800 & 0.0700 & 100 \\
\hline & 10 & 0.8800 & 0.4200 & 0.4800 & 3 \\
\hline \multirow{3}{*}{17} & 10 & 0.0200 & 0.1100 & 0.1000 & 100 \\
\hline & 10 & 3.3600 & 1.2600 & 2.4800 & 3 \\
\hline & 10 & 0.0400 & 0.0900 & 0.0800 & 100 \\
\hline \multirow[t]{2}{*}{18} & 10 & 0.7200 & 0.4500 & 0.4000 & 4 \\
\hline & 10 & 0.0100 & 0.1400 & 0.1000 & 100 \\
\hline \multirow[t]{2}{*}{20} & 10 & 4.2300 & 1.3700 & 1.1900 & 6 \\
\hline & 10 & 40.8700 & 28.5900 & 37.5400 & 4 \\
\hline \multirow{3}{*}{22} & 10 & 0.0900 & 0.3400 & 0.2200 & 100 \\
\hline & 10 & 119.8300 & 24.4700 & 41.4500 & 10 \\
\hline & 10 & 318.6700 & 111.0000 & 161.4000 & 8 \\
\hline \multirow{3}{*}{24} & 10 & 0.2000 & 0.3700 & 0.3400 & 100 \\
\hline & 10 & 495.2300 & 72.7200 & 64.9700 & 8 \\
\hline & 10 & 5152.6200 & 2021.1300 & 2737.3300 & 6 \\
\hline \multirow{3}{*}{25} & 10 & 0.0700 & 0.3600 & 0.2000 & 100 \\
\hline & 10 & 71.9100 & 7.1500 & 13.6000 & 12 \\
\hline & 10 & 197.0500 & 88.7900 & 84.1000 & 10 \\
\hline \multirow{3}{*}{27} & 10 & 0.3700 & 0.7400 & 0.3400 & 100 \\
\hline & 10 & 1082.2300 & 61.5200 & 48.4700 & 13 \\
\hline & 10 & 9144.4700 & 1027.0800 & 1990.6200 & 10 \\
\hline \multirow[b]{2}{*}{28} & 10 & 0.8600 & 1.5300 & 0.4000 & 100 \\
\hline & 10 & 157.9300 & 30.1700 & 28.9200 & 10 \\
\hline \multirow{3}{*}{30} & 10 & 0.2800 & 0.7100 & 0.7300 & 100 \\
\hline & 10 & 553.2400 & 41.7000 & 52.2900 & 13 \\
\hline & 10 & 42142.2800 & 8631.8100 & 8830.0700 & 10 \\
\hline \multirow{3}{*}{40} & 10 & 0.2700 & 7.2800 & 0.9800 & 100 \\
\hline & 10 & 8757.5500 & 908.6300 & 966.8900 & 14 \\
\hline & 10 & 0.9700 & 28.3600 & 3.4800 & 100 \\
\hline \multirow[t]{2}{*}{50} & 10 & 295.2800 & 56.6600 & 34.8500 & 25 \\
\hline & 10 & 16.3500 & 2839.4700 & 106.8900 & 1000 \\
\hline \multirow[t]{2}{*}{100} & 10 & 110446.7300 & 6804.7700 & 700.3100 & 35 \\
\hline & 10 & 5.2000 & 34421.1500 & 298.7300 & 1000 \\
\hline 150 & 10 & 249377.7300 & 154887.6300 & 5348.4600 & 60 \\
\hline
\end{tabular}

Na tabela 4, nota-se que Cplex se mostra muito rápido nas capacidades maiores e nas instâncias menores, mas a medida que a capacidade diminui e as instâncias os outros algoritmos se mostram mais eficientes. Em relação as formulações de corte de Benders agregado e desagregado que conforme Magnanti et al. (1986) as formulações são mais eficientes do que as obtidas com as restrições de agregação, percebe-se que se o número de capacidade for menor e o número de nós da instância for maior é mais útil trabalhar com os cortes de desagregados, caso contrário, é melhor trabalhar com cortes agregados.

\subsection{Análise dos Resultados}

Dos resultados obtidos pode-se ver que o CPLEX é boa ferramenta para resolver o problema em estudo, porém não a mais rápida. Sendo assim, é melhor resolvê-lo com uma única árvore ao invés de utilizar a implementação tradicional da Decomposição de Benders.

$\mathrm{O}$ método $\lambda$-ótimo, assim como o esquema $\mathrm{OA}+\mathrm{BD}$ se mostraram as ferramentas mais rápidas e precisas para esta importante classe de problemas. É possível perceber que as instâncias ficam mais difíceis de resolver quando o custo de congestionamento aumenta. 
As figuras 1 e 2 exemplificam o resultado do problema mostrando que, para uma mesma instância, há uma diferença nas conexões instaladas na rede ótima.

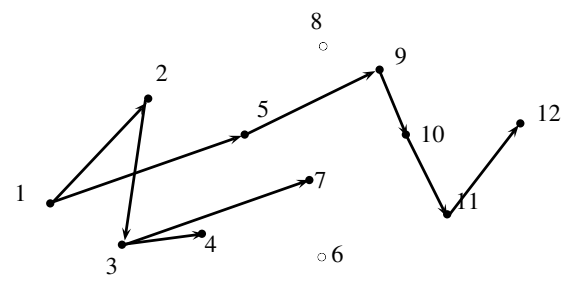

Figura 1: Solução sem congestionamento.

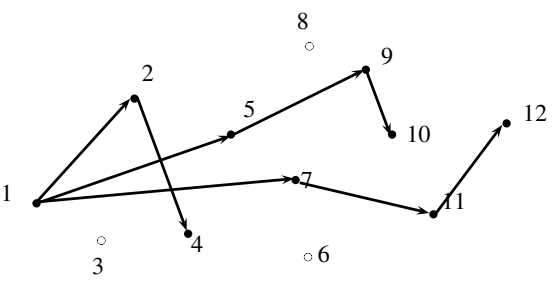

Figura 2: Solução com congestionamento.

\section{Conclusão}

Neste trabalho aplicou-se a técnica de seleção de cortes de Benders conhecida como $\lambda$ ótimo ao problema de projeto de redes sob congestionamento, atacado via Aproximação Externa/ Decomposicão de Benders (OA+BD). Os conjuntos de experimentos demostram que o custo do congestionamento em cada instância afetam o tempo de resolução de cada algoritmo. De acordo com os testes analisa-se que a medida que aumenta o custo de congestionamento aumenta também o esforço computacional para resolver o problema em estudo.

Estudos impirícos confirmaram que a variante do OA+BD via $\lambda$-ótimo apresenta a melhor performace entre as demais testadas, ou seja, esta variante resolve o problema proposto em menor tempo computacional e é capaz de resolver instâncias maiores que os demais analisados. Esta combinação de técnicas é promissora para a solução de grandes instância deste importante problema. Este algoritmo é capaz de resolver a maioria das instâncias significativamente mais rápido do que o CPLEX 12 na formulação original. Pode-se ver também, que os cortes de Benders agregado são bastante competitivos, mas medida que a instância aumenta torna-se mais eficaz utilizá-los desagregados.

\section{Referências}

Altiparmak, F., Dengiz, B., and Smith, A. E. (2003). Optimal design of reliable computer networks: A comparison of metaheuristics. Journal of Heuristics, 9(6):471-487.

Benchakroun, A., Ferland, J., and Cleroux, R. (1992). Distribution system planning through a generalized benders decomposition approach. European journal of operational research, 62(2):149162.

Contreras, I., Fernández, E., and Marín, A. (2009). Tight bounds from a path based formulation for the tree of hub location problem. Computers \& Operations Research, 36(12):3117-3127.

Contreras, I., Fernández, E., and Marín, A. (2010). The tree of hubs location problem. European Journal of Operational Research, 202(2):390-400.

Cordeau, J.-F., Pasin, F., and Solomon, M. M. (2006). An integrated model for logistics network design. Annals of operations Research, 144(1):59-82.

Costa, A. M. (2005). A survey on benders decomposition applied to fixed-charge network design problems. Computers \& operations research, 32(6):1429-1450.

Dantzig, G. (1962). Linear Programming and Extensions. Princeton University Press.

De Camargo, R. S., De Miranda, G., and Ferreira, R. P. (2011). A hybrid outerapproximation/benders decomposition algorithm for the single allocation hub location problem under congestion. Operations Research Letters, 39(5):329-337. 
de Sá, E. M., de Camargo, R. S., et al. (2013). An improved benders decomposition algorithm for the tree of hubs location problem. European Journal of Operational Research.

Dias, P. G. F., de Miranda Jr, G., Saldanha, R. R., and de Camargo, R. S. (2011). Projeto de rede com custos convexos e balanceamento de fluxos. Revista Controle \& Automação/Vol. X no. $X / F e v$, page 1 .

Duran, M. and Grossmann, I. E. (1986). An outer-approximation algorithm for a class of mixed integer nonlinear programms. Mathematical Programming, 36:307-339.

Fletcher, R. and Leyffer, S. (1994). Solving mixed integer nonlinear programs by outer approximation. Mathematical programming, 66(1-3):327-349.

Geoffrion, A. (1972). Generalized Benders decomposition. Journal of optimization Theory and Applications, 10(4):237-260.

Grossmann, I. E. and Kravanja, Z. (1995). Mixed-integer nonlinear programming techniques for process systems engineering. Computers \& chemical engineering, 19:189-204.

Hahn, P., Anjos, M., Burkard, R., Karisch, S., and Rendl, F. (2006). Qaplib-a quadratic assignment problem library. Avialable at http://www. seas. upenn. edu/qaplib.

Huang, S., Batta, R., and Nagi, R. (2005). Distribution network design: Selection and sizing of congested connections. Naval Research Logistics (NRL), 52(8):701-712.

Hwang, F. K. and Richards, D. S. (1992). Steiner tree problems. Networks, 22(1):55-89.

Karuppiah, R., Furman, K. C., and Grossmann, I. E. (2008). Global optimization for scheduling refinery crude oil operations. Computers \& Chemical Engineering, 32(11):2745-2766.

Klincewicz, J. G. (1998). Hub location in backbone/tributary network design: a review. Location Science, 6:307-335.

Maculan, N. (1987). The Steiner problem in graphs. Annals of Discrete Mathematics, 31:185-212.

Magnanti, T. L., Mirchandani, P., and Wong, R. T. (1986). Tailoring Benders decomposition for uncapacitated network design. Mathematical Programming Study, 26:112-154.

Magnanti, T. L. and Wong, R. T. (1981). Accelerating benders decomposition: Algorithmic enhancement and model selection criteria. Operations Research, 29(3):464-483.

Miranda, G., Luna, H., De Camargo, R., and Pinto, L. (2011). Tree network design avoiding congestion. Applied Mathematical Modelling, 35(9):4175-4188.

Papadakos, N. (2008). Practical enhancements to the magnanti-wong method. Operations Research Letters, 36:444-449.

Ramírez-Rosado, I. J. and Domínguez-Navarro, J. A. (2006). New multiobjective tabu search algorithm for fuzzy optimal planning of power distribution systems. Power Systems, IEEE Transactions on, 21(1):224-233.

Randazzo, C. and Luna, H. (2001). A comparison of optimal methods for local access uncapacitated network design. Annals of Operations Research, 106:263-286.

Yuan, X., Zhang, S., Pibouleau, L., and Domenech, S. (1988). Une méthode d'optimisation non linéaire en variables mixtes pour la conception de procédés. RAIRO-Operations ResearchRecherche Opérationnelle, 22(4):331-346. 